\title{
Can pharmacogenomics guide effective anticancer therapy in pancreatic ductal adenocarcinoma?
}

\author{
“...can we really celebrate our successes in improving treatments for pancreatic \\ ductal adenocarcinoma in the gemcitabine era?"
}

\section{KEYWORDS: gemcitabine - pancreatic cancer " pharmacogenomics $\approx$ resistance}

The development of new targeted therapies has become a major goal in the modern era of cancer research and treatment and has provided a significant stimulus for the evolving field of pharmacogenomics. However, we must not forget that tamoxifen, the first targeted anticancer agent, was initially developed and used in breast cancer in the early 1970s, but some 30 years elapsed before the emergence of the next major targeted therapy, trastuzumab. In contrast to the initially slow progress in targeted therapy design during this period, there was significant success in the development or reinvention of 'nontargeted' chemotherapeutic agents and chemotherapy regimens through large Phase II and Phase III studies. However the design of such drugs and combination regimens has not always resulted in significant improvements in cancer patient care and survival. Much of this must be attributed to a lack of understanding of the role of pharmacogenomics in both response and toxicity associated with these agents, and there are few better examples of this than gemcitabine in pancreatic ductal adenocarcinoma (PDAC). Furthermore, despite the significant advances over the last decade in the development of molecularly targeted therapies in many human cancers, the impact of novel agents on clinical outcomes in PDAC patients has been lacking.

5-fluorouracil (5-FU) was the recognized treatment for advanced PDAC until 1997, but following an observed improvement in clinical benefit (determined by pain scores and analgesic requirements), and overall survival (OS) in a randomized comparison with 5-FU, gemcitabine was established as the standard of care for treating this disease [1]. Subsequently trialed chemotherapy regimens, that often included gemcitabine, have had variable success but often failed to demonstrate an OS benefit over gemcitabine alone. A large Phase III study comparing gemcitabine with gemcitabine plus capecitabine (gem-cap), did demonstrate a significant benefit in objective response rate and progression-free survival with the combination regimen but only showed a trend for improvement in OS, although a subsequent meta-analysis of two additional studies involving 935 patients showed a significant survival benefit in favor of gem-cap (hazard ratio: 0.86 ; $95 \% \mathrm{CI}$ : $0.75-0.98 ; p=0.02$ ) [2]. Others have shown increased response rates but often at the expense of increased toxicity. FOLFIRINOX represents the most recent significant therapeutic advance in PDAC, as it improved survival in good performance status patients with metastatic disease by 4.3 months compared with gemcitabine alone [3], but again that came at the cost of significantly increased toxicities, in particular, hematological and gastrointestinal. Clinical trials involving multiple targeted agents acting on various cellular signaling pathways have also had variable success in terms of improving outcome measures. Erlotinib, which targets the EGF receptor, is potentially an exception to this as its use in combination with gemcitabine in patients with advanced disease was associated with a significant survival benefit (median 6.24 vs 5.91 months; $\mathrm{p}=0.038$ ) compared with gemcitabine alone [4], although some would question the clinical significance of these findings. We also await the results of a Phase II study of a cytotoxic doublet/biologic doublet regimen involving gem-cap in combination with bevacizumab and erlotinib in advanced pancreatic cancer patients [5]. Furthermore in a recent Phase II study, gemcitabine plus nab-paclitaxel was well tolerated and associated with a response rate of $48 \%$, a median OS of 12.2 months and a $48 \% 1$-year survival, and we now await a Phase III evaluation [6].

So can we really celebrate our successes in improving treatments for PDAC in the gemcitabine era? One would have to argue that we cannot. After all, the 5-year survival rates for

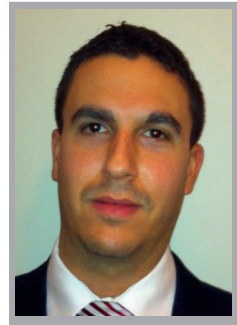

Jonathan Krell

Author for correspondence: Division of Cancer, Department of

Surgery \& Cancer, 5th Floor, MRC Cyclotron Building, Imperial College, Hammersmith Hospital, Du Cane Road, London, W12 ONN, UK j.krell@imperial.ac.uk

Adam E Frampton

HPB Surgical Unit, Department of Surgery \& Cancer, Imperial College, Hammersmith Hospital, Du Cane Road, London, W12 OHS, UK

\section{Long R Jiao}

HPB Surgical Unit, Department of Surgery \& Cancer, Imperial College, Hammersmith Hospital, Du Cane Road, London, W12 OHS, UK

\section{Justin Stebbing}

Department of Oncology, Imperial College Healthcare NHS Trust, Charing Cross Hospital, 1st Floor, E Wing, Fulham Palace Road, London, W6 8RF, UK

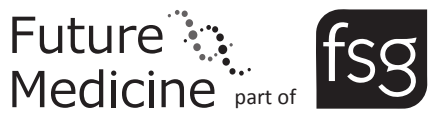


advanced PDAC remain under 5\% and arguably the greatest advance in chemotherapeutic strategy for treating this disease involves a combination regimen, FOLFIRINOX, which was associated with a median OS in good performance patients of only 11.1 months [3]. One cannot ignore the fact that this is nearly double the median OS seen in patients treated with gemcitabine alone, but although FOLFIRINOX therapy was also associated with significantly lower levels of degradation in quality of life at 6 months [3], the increased side effects associated with FOLFIRINOX when compared with gemcitabine should also not be understated. Surely throwing more and more chemotherapies into the equation is not necessarily the approach to adopt and one strategy in improving outcome in PDAC should be to better understand which patients benefit most from current therapies and to potentially use synergistic agents to maximize response. We believe that a better understanding of the pharmacogenomics associated with the efficacy and toxicity of gemcitabine holds the key to achieving this.

"...the 5-year survival rates for advanced pancreatic ductal adenocarcinoma remain under 5\% and arguably the greatest advance in chemotherapeutic strategy for treating this disease involves a combination regimen, FOLFIRINOX, which was associated with a median overall survival in good performance patients of only 11.1 months."

Gemcitabine is a deoxycytidine analog and is administered as a prodrug that requires cellular uptake and intracellular phosphorylation to exert its action. Cellular uptake involves two families of nucleoside transporters, the sodiumdependent hENT1 and 2 equilibrative transporters, and the sodium-independent hCNT 1-3 concentrative transporters [7]. Following transport into cells gemcitabine is phosphorylated by DCK to form gemcitabine monophosphate, which is subsequently phosphorylated to gemcitabine diphosphate and gemcitabine triphosphate (dFdCTP) by nucleoside monophosphate (uridine monophosphate/cytidine monophosphate) and diphosphate kinase. Gemcitabine exerts its cytotoxic effect mainly through inhibition of DNA synthesis via the incorporation of active dFdCTP into the DNA strand [7]. It also has a unique mechanism of action known as 'self-potentiation' [8]. However, over $90 \%$ of administered gemcitabine is inactivated by cytidine deaminase which converts active dFdCTP into inactive $2^{\prime}$-deoxy-2', $2^{\prime}$-difluorouridine. Additionally, phosphorylated gemcitabine metabolites are reduced by cellular 5'-nucleotidase, and gemcitabine monophosphate is also inactivated by deoxycytidine monophosphate deaminase [7]. Therefore, pharmacogenomics must play a key role in the interpatient variability of gemcitabine's antitumor activity and toxicity, as the complex pathways involved in its metabolism and mechanism of action provide several key stages that may be affected by an increase or decrease in the activity of one or more of these components.

“...recent progress towards understanding the genetic events in the development of pancreatic ductal adenocarcinoma, in combination with advances in the field of pharmacogenomics, offer hope that we may build on achievements to date to better utilize standard treatments such as gemcitabine, as well as to develop more effective therapeutic strategies for pancreatic ductal adenocarcinoma in the future."

A number of studies have correlated tumor hENT levels with clinical outcomes in PDAC patients and results suggest a lack of clinical benefit to the use of gemcitabine in those with absent or low hENT1 levels, although this hypothesis requires prospective validation $[9,10]$. There is significant variability in DCK expression levels between patients carrying different $D C K$ genotypes [11] and in a study of 44 patients with advanced PDAC, reduced expression of DCK correlated with reduced OS and progression-free survival following gemcitabine-based chemotherapy (14.6 vs 21.7 months; $\mathrm{p}<0.04$ ) [12]. Others have shown that pharmacogenomic variation in the deamination of gemcitabine and its monophosphate might contribute to variation in therapeutic response to this [13]. Furthermore, genetic polymorphisms of various DNA damage and repair genes can have significant effects on outcome following gemcitabine based chemotherapy [14].

PDAC has historically been very challenging to treat with standard chemotherapy and the outcome for most patients is usually very poor. Although a number of common mutations have been identified as playing a significant role in the development and progression of PDAC, the reasons behind this poor outcome and response to therapy is unclear. Clinically not all PDACs behave in the same way. Relapse rates for patients diagnosed with early stage disease are high, but some patients do not and the reasons for this are 
unclear. Furthermore, we see significant variability in terms of response and toxicity to gemcitabine therapy in PDAC patients, with objective response rates in the region of $10-15 \%$ in advanced disease. Many patients who do respond can do so for a prolonged period of time and to these patients, gemcitabine is often very beneficial. However, the majority of patients do not obtain any significant benefit from gemcitabine monotherapy. Although these differences may relate to variability in the expression of oncogenes and tumor suppressor genes that drive tumor growth, it appears likely that pharmacogenomic factors must also play an important role. It therefore seems illogical that in an age when targeted therapies and the identification of prognostic and predictive biomarkers are the vogue, we are still unable to use similar methods to routinely identify which patients with PDAC are most likely to benefit from gemcitabine chemotherapy and therefore should receive the drug, and which patients may benefit more from an alternative regimen. Although the nongemcitabine-containing regimen, FOLFIRINOX has been shown to be superior to single agent gemcitabine in terms of response rates and survival it is associated with increased toxicity. Therefore, a greater understanding of the pharmacogenomics of gemcitabine and 5-FU, irinotecan and oxaliplatin would enable physicians to better select which patients are most likely to respond effectively to which regimen, and also determine the likely toxicities. This is obviously not an easy task but there should be sufficient tumor tissue from previous and current studies to both retrospectively and prospectively investigate this further.

Certainly, the challenge remains to develop more effective, less toxic systemic therapies against PDAC, and this may well lie in the design of novel targeted therapies. However, recent progress towards understanding the genetic events in the development of PDAC, in combination with advances in the field of pharmacogenomics, offer hope that we may build on achievements to date to better utilize standard treatments such as gemcitabine, as well as to develop more effective therapeutic strategies for PDAC in the future.

\section{Financial \& competing interests disclosure}

The authors have no relevant affliations or financial involvement with any organization or entity with a financial interest in or financial conflict with the subject matter or materials discussed in the manuscript. This includes employment, consultancies, honoraria, stock ownership or options, expert testimony, grants or patents received or pending, or royalties.

No writing assistance was utilized in the production of this manuscript.

\section{References}

1 Burris HA 3rd, Moore MJ, Andersen J et al. Improvements in survival and clinical benefit with gemcitabine as first-line therapy for patients with advanced pancreas cancer: a randomized trial. J. Clin. Oncol. 15(6), 2403-2413 (1997).

2 Cunningham D, Chau I, Stocken DD et al. Phase III randomized comparison of gemcitabine versus gemcitabine plus capecitabine in patients with advanced pancreatic cancer. J. Clin. Oncol. 27(33), 5513-5518 (2009).

3 Conroy T, Desseigne F, Ychou M et al. FOLFIRINOX versus gemcitabine for metastatic pancreatic cancer. N. Engl. J. Med. 364(19), 1817-1825 (2011).

4 Moore MJ, Goldstein D, Hamm J et al. Erlotinib plus gemcitabine compared with gemcitabine alone in patients with advanced pancreatic cancer: a Phase III trial of the National Cancer Institute of Canada Clinical Trials Group. J. Clin. Oncol. 25(15), 1960-1966 (2007).

5 Starling N, Watkins D, Cunningham D et al. Dose finding and early efficacy study of gemcitabine plus capecitabine in combination with bevacizumab plus erlotinib in advanced pancreatic cancer. J. Clin. Oncol. 27(33), 5499-5505 (2009).

6 Von Hoff DD, Ramanathan RK, Borad MJ et al. Gemcitabine plus nab-paclitaxel is an active regimen in patients with advanced pancreatic cancer: a Phase I/II trial. J. Clin. Oncol. 29(34), 4548-4554 (2011).

7 Ueno H, Kiyosawa K, Kaniwa N. Pharmacogenomics of gemcitabine: can genetic studies lead to tailor-made therapy? Br. J. Cancer. 97(2), 145-151 (2007).

8 Heinemann V, Xu YZ, Chubb S et al. Cellular elimination of

2',2'-difluorodeoxycytidine 5'-triphosphate: a mechanism of self-potentiation. Cancer Res. 52(3), 533-539 (1992).

9 Regine WF, Winter KA, Abrams R et al. Fluorouracil-based chemoradiation with either gemcitabine or fluorouracil chemotherapy after resection of pancreatic adenocarcinoma: 5-year analysis of the U.S. Intergroup/RTOG 9704 Phase III trial. Ann. Surg. Oncol. 18(5), 1319-1326 (2011).
10 Farrell JJ, Elsaleh H, Garcia M et al. Human equilibrative nucleoside transporter 1 levels predict response to gemcitabine in patients with pancreatic cancer. Gastroenterology 136(1), 187-195 (2009).

11 Shi JY, Shi ZZ, Zhang SJ et al. Association between single nucleotide polymorphisms in deoxycytidine kinase and treatment response among acute myeloid leukaemia patients. Pharmacogenetics 14(11), 759-768 (2004).

12 Sebastiani V, Ricci F, Rubio-Viqueira B et al. Immunohistochemical and genetic evaluation of deoxycytidine kinase in pancreatic cancer: relationship to molecular mechanisms of gemcitabine resistance and survival. Clin. Cancer Res. 12 (8), 2492-2497 (2006).

13 Gilbert JA, Salavaggione OE, Ji Y et al. Gemcitabine pharmacogenomics: cytidine deaminase and deoxycytidylate deaminase gene resequencing and functional genomics. Clin. Cancer Res. 12(6), 1794-1803 (2006).

14 Li D, Frazier M, Evans DB et al. Single nucleotide polymorphisms of RecQ1, $R A D 54 L$, and $A T M$ genes are associated with reduced survival of pancreatic cancer. J. Clin. Oncol. 24(11), 1720-1728 (2006). 